\title{
Spiral computed tomography angiography in the assessment of middle cerebral artery occlusive disease
}

\author{
K S Wong, E Y Liang, W W M Lam, Y N Huang, R Kay
}

\begin{abstract}
There has been no report on the use of spiral computed tomography angiography (CTA) in the investigation of intracranial cerebral artery stenosis. A prospective pilot study was conducted to investigate the feasibility of CTA in the diagnosis of intracranial occlusive disease and its correlation with transcranial Doppler. With transcranial Doppler, 10 patients with acute ischaemic stroke with middle cerebral artery stenosis or occlusion were identified. There were seven middle cerebral artery stenoses and five middle cerebral artery occlusions. The CTA confirmed all diagnoses by transcranial Doppler except in one patient with middle cerebral artery occlusion in whom the embolus had probably propagated. The results showed that CTA is feasible and potentially useful in the diagnosis of middle cerebral artery occlusive disease. Further studies are required to assess its validity, sensitivity, and specificity in the diagnosis of middle cerebral artery occlusive disease.
\end{abstract}

(F Neurol Neurosurg Psychiatry 1995;59:537-539)

Department of

Medicine

K S Wong

R Kay

Department of

Diagnostic Radiology

and Organ Imaging,

The Chinese

University of Hong

Kong, Prince of Wales

Hospital, Shatin,

Hong Kong

E Y Liang

W W M Lam

Department of

Neurology, Peking

Union Medical

College Hospital,

Beijing, China

Y N Huang

Correspondence to:

Dr K S Wong, Department

of Medicine, Prince of

Wales Hospital, The

Chinese University of Hong

Kong, Shatin, Hong Kong.

Received 29 March 1995

and in revised form

and in revised

Accepted 23 June 1995
Keywords: computed tomography angiography; cerebral infarction; intracranial stenosis.

Assessment of the middle cerebral artery is an important investigation for patients with stroke, especially in the populations in whom intracranial atherosclerosis is a common cause of stroke. Currently available techniques in clinical practice include conventional angiography, transcranial Doppler, and magnetic resonance angiography. Spiral CT angiography (CTA) is a new, fast, and minimally invasive technique that is able to provide images of the blood vessel with sufficient clarity to quantify the degree of stenosis. ${ }^{1}$ Although recent studies have shown that it is a sensitive and specific technique for carotid stenosis $^{2}$ and intracranial aneurysm, ${ }^{3}$ there is no published report on the use of CTA in the investigation of patients with stroke with intracranial occlusive disease. We conducted a pilot study of CTA in patients with acute stroke and assessed whether the findings cor- related with the findings from transcranial Doppler in the assessment of middle cerebral artery occlusive disease.

\section{Methods}

We identified 10 patients with middle cerebral artery stenosis or occlusion by transcranial Doppler among patients who presented to a regional hospital with acute stroke. Transcranial Doppler was performed with a TC2000 EME machine (Uberlingen, Germany) according to standard procedures. ${ }^{4}$ Extracranial carotid arteries were examined by a $4 \mathrm{mHz}$ probe to exclude significant stenosis. We diagnosed middle cerebral artery stenosis if its peak systolic velocity was greater than $140 \mathrm{~cm} / \mathrm{s}^{5}$ and middle cerebral artery occlusion if all basal arteries except the middle cerebral artery in question were detectable $^{6}$ or if there was a more than $21 \%$ reduction of its mean velocity compared with that of the contralateral middle cerebral artery. ${ }^{7}$ Significant extracranial carotid stenosis was diagnosed if its peak systolic velocity was higher than $120 \mathrm{~cm} / \mathrm{s}$ and the systolic ratio was more than $1 \cdot 8$.

Spiral CT angiography was performed within 24 hours of the Doppler examination. The procedure was performed with a General Electric Hispeed Advantage scanner (General Electric Medical Systems, Milwaukee, WI, USA). A $20 \mathrm{G}$ or larger intravenous catheter was placed into an antecubital vein. A dose of $135 \mathrm{ml}$ of iohexol (Omnipaque 300, Sanofi Winthrop Pharmaceuticals) was prepared, to be given at the rate of $3 \mathrm{ml} / \mathrm{s}$ through a power injector. A lateral scout view was then performed. The gantry was tilted parallel to the floor of the anterior cranial fossa. A starting point was selected at the floor of the sella. Helical scanning of 35 second continuous exposure ( $1 \mathrm{~mm}$ collimation, $1 \mathrm{~mm} / \mathrm{s}$ table speed, $120 \mathrm{kVp}, 240-280 \mathrm{~mA}$ ) was started 20 seconds after commencement of contrast injection. Raw data were interpolated into 35 $1 \mathrm{~mm}$ axial images on the scanner. To improve resolution of vessels of the circle of Willis, these images were then reconstructed using a display field of view of 9.6 to $10.0 \mathrm{~cm}$. This also facilitated the removal of temporal bones and yet included the middle cerebral artery bifurcation with some of its 
branches. The reconstructed axial images were then transferred to a work station (Advantage Window, General Electric Medical System, Milwaukee WI, USA). The

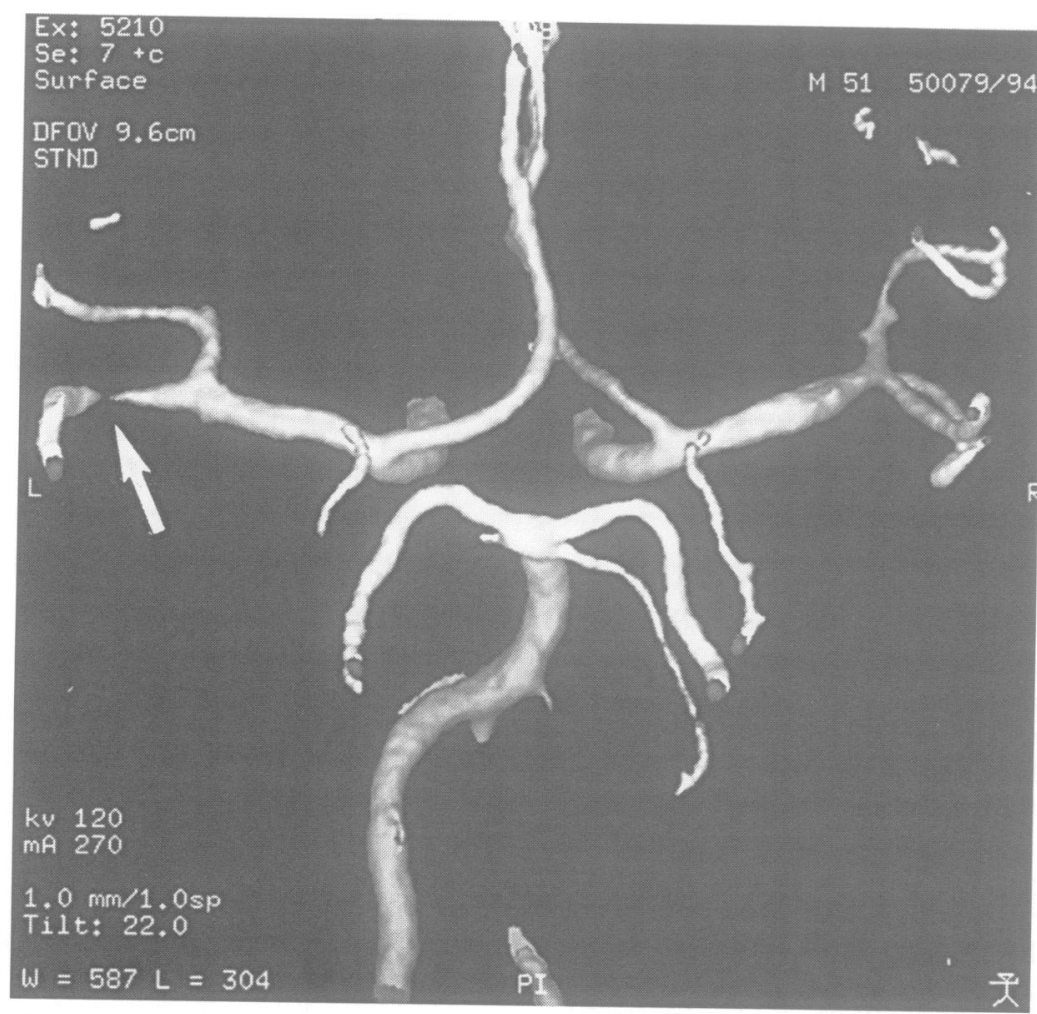

A

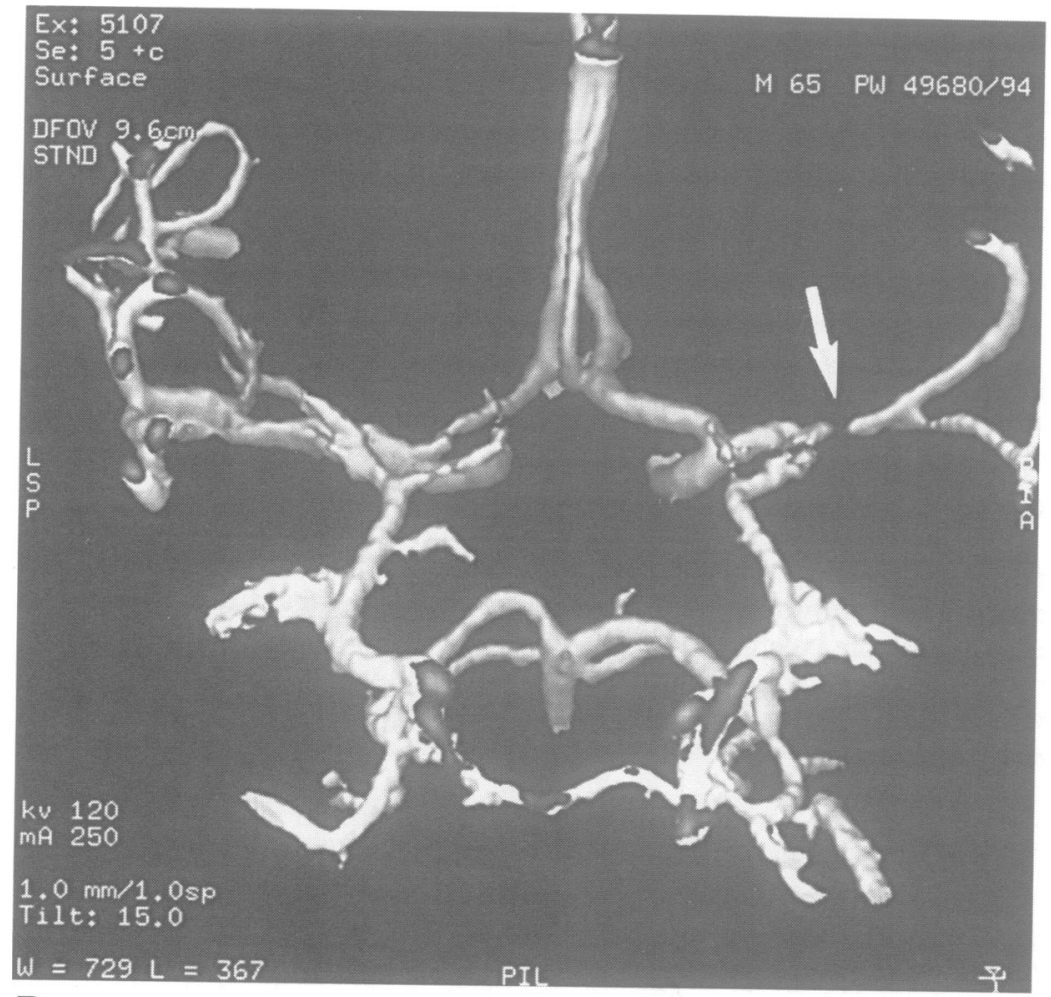

B

Computed tomography angiography of the basal arteries. (A) stenosis of a branch of the left middle cerebral artery (arrow); (B) occlusion of the right left middle cerebral artery
stem (arrow).
CTAs were read "blind" to the transcranial Doppler findings.

\section{Results}

All transcranial Doppler and CTA studies were performed within three days of the symptomatic stroke. A total of 10 patients were studied and all patients had had significant hemiparesis. There were no complications attributable to contrast medium in our patients. Transcranial Doppler showed seven middle cerebral artery stenoses and five middle cerebral artery occlusions. In all seven stenoses, CTA correlated well with transcranial Doppler. In one patient, transcranial Doppler suggested a left distal middle cerebral artery stenosis (the depth of the increased velocity was $32 \mathrm{~mm}$ ); CTA showed a severe stenosis at the corresponding site (fig $1 \mathrm{~A})$. In the remaining patients with middle cerebral artery stenoses, CTA showed stenosis in the middle cerebral artery stems and this also correlated well with the Doppler findings.

In five patients with transcranial Doppler diagnoses of middle cerebral artery occlusion, CTA showed middle cerebral artery occlusions in four (fig 1B). In the remaining patient, CTA was performed 18 hours after transcranial Doppler. No occlusive disease was shown on CTA but a repeat Doppler performed immediately after CTA showed normalised velocity. These findings suggested that the middle cerebral artery occlusion may have been caused by an embolus that was dislodged more distally before the CTA examination.

\section{Discussion}

Although carotid occlusive disease is an important cause of ischaemic stroke, intracranial atherosclerosis is more common than extracranial atherosclerosis in Chinese, Japanese, and black people. ${ }^{89}$ Conventional angiography is regarded as the gold standard for investigation of the middle cerebral artery. This procedure is, however, invasive and associated with significant morbidity. Transcranial Doppler is a non-invasive and sensitive method to study middle cerebral artery occlusive diseases. ${ }^{10}$ It provides additional information on the haemodynamics of the cerebral circulation. In up to $35 \%$ of patients with stroke (depending on age and sex), however, there is insufficient ultrasound penetration through the temporal bone and it is impossible to perform transcranial Doppler through the temporal window. ${ }^{11}$

Spiral CT angiography is a new, minimally invasive technique. Its advantages include short examination time, clarity of arterial calibre in areas of stenosis, and it is probably more accessible than magnetic resonance angiography. CTA can be performed at the same time as the routine CT of patients with acute stroke. According to our protocol, CTA requires 35 seconds of scanning time and thus is particularly suitable for patients who 
are confused, unstable, or claustrophobic. Therefore, CTA may be a useful alternative for the investigation of middle cerebral artery stenosis.

The disadvantages of CTA are the problems associated with contrast injection (in patients with cardiac failure and allergy) and only limited segments of the cerebral vasculature can be imaged at the same time. The use of ionic contrast medium in patients with acute stroke is associated with poor functional recovery. Modern non-ionic contrast media are, however, relatively safe and do not cross the blood-brain barrier. ${ }^{12}$

In this study, CTA provides no further information than transcranial Doppler except better visualisation of the vessels. Thus these two techniques are complementary. The main findings of our study were that CTA correlated well with transcranial Doppler in diagnosing middle cerebral artery stenosis, and the clarity of middle cerebral artery stenosis as it appeared in CTA made quantification of stenosis a possibility.

In conclusion, our preliminary experience showed that CTA is feasible and it correlates well with transcranial Doppler in the diagnosis of middle cerebral artery occlusive disease. Further studies are required to assess its validity, sensitivity, and specificity in the investigation of intracranial cerebral artery stenosis before CTA can be routinely used in clinical practice.
This study was supported by Direct Grant for Research 94/95 No 220403890, Research Grant Committee, the Chinese University of Hong Kong, Hong Kong.

1 Schwartz RB. Neuroradiological applications of spiral CT. Seminars in Ultrasound, CT, and MR. Magn Reson Imaging 1994;15:139-47.

2 Cumming MJ, Morrow IM. Carotid artery stenosis: a prospective comparison of CT angiography and conventional angiography. $A \mathcal{F} R A m \mathcal{F}$ Roentgenol 1994;163: 517-23.

3 Schwartz RB, Tice HM, Hooten SM, Hsu RTL, Stieg PE. Evaluation of cerebral aneurysms with helical CT; correlation with conventional angiography and $M R$ angiography. Radiology 1994;192:717-22.

4 Hennerici M, Mohr JP, Rautenberg W, Steinke W. Ultrasound imaging and Doppler sonography in the diagnosis of cerebrovascular diseases. In Barnett $\mathrm{HJM}$, Mohr JP, Stein BM, Yatsu FM, eds. Stroke: pathophysiMohr JP, Stein BM, Yatsu FM, eds. Stroke: pathophysiology, diagnosis, and managem

5 Rother J, Schwartz A, Wentz KU, Rautenberg W, Rother J, Schwartz A, Wentz KU, Rautenberg W,
Hennerici M. Middle cerebral artery stenosis: assessment by magnetic resonance angiography and transcranial Doppler ultrasound. Cerebrovasc Dis 1994;4: 273-9

6 Kaps M, Damian MS, Teschendorf U, Dorndorf W. Transcranial Doppler ultrasound findings in middle cerebral artery occlusion. Stroke 1990;21:532-7.

7 Zanette EM, Fieschi C, Bozzao L, Roberti C, Toni D, Argentino C, Lenzi GL. Comparison of cerebral angiography and transcranial Doppler sonography in acute stroke. Stroke 1989;20:899-903.

8 Leung SY, Ng THK, Yuen ST, Lauder IJ, Ho FCS. Pattern of cerebral atherosclerosis in Hong Kong Chinese: severity in intracranial and extracranial vessels. Stroke 1993;24:779-86.

9 Caplan LR, Gorelick PB, Hier DB. Race, sex and occluaplan LR, Gorelick PB, Hier DB. Race, sex and occlu-
sive cerebrovascular diseases: a review. Stroke 1986;17: 648-55.

10 Kushner MJ, Zanette EM, Bastianello S, Mancini G, Sacchetti ML, Carolei A, Bozzao L. Transcranial Doppler in acute hemispheric brain infarction. Neurology 1991;41:109-13.

11 Laps $M$, Teschendorf $U$, Dorndorf $W$. Haemodynamic studies in early stroke. $\mathcal{F}$ Neurol 1992;239:138-42.

12 Velaj R, Drayer B, Albright R, Fram E. Comparative neurotoxicity of angiographic contrast media. Neurology 1985;35:1290-8. 